\title{
Editorial
}

\section{Changes to the Instructions for Authors}

The New Year brings with it fresh opportunity for us all. In this spirit we thought it timely to write an editorial both to explain some of our concerns about the submissions we receive for the Journal, and to emphasize changes that we have made in the Instructions for Authors - which have been updated and which are published in revised format for the first time in this edition. All medical and scientific journals set down instructions to authors - which are necessary to ensure uniformity of style and to facilitate the editorial and publication process. In spite of this we continue to receive a significant number of manuscripts which fail because these guidelines have been ignored. It is our hope that the highlighting of these problems, and the changes that we have made, will be seen in an constructive light and we trust that by clarifying certain issues we will be able to reduce unnecessary delay in publishing submitted material.

\section{Manuscripts and illustrations}

Even the simplest instructions are frequently disregarded. Thus we still receive contributions which are single spaced and which lack reasonable margins. The same lack of attention to detail applies to referencing: many papers are submitted where references are either cited in the text and then fail to appear in the list at the end of the paper (or vice versa) or are not listed in alphabetical order (by name of first authors). Manuscripts referenced in the Vancouver rather than the Harvard system are also frequently submitted - which stir some of our more cynical reviewers into believing that the paper has already been rejected by another journal! With the widespread use of word processors it is difficult to excuse such elementary oversights. There is also concern about the quality of the illustrations which we receive. Illustrations should not exceed $80 \mathrm{~mm}$ width and should be of good quality. It goes without saying that they should also unequivocally illustrate the features that the authors are trying to demonstrate! Routine photomicrographs of pathological specimens also pose problems: they should include details of the staining technique that has been employed and the magnification at which the preparation has been photographed. Illustrations which do not satisfy these requirements are not acceptable.

\section{Key words}

The concept behind key words is clearly remote to the majority. Some papers are received without any attempt at providing these, but the majority simply contain words which the authors have seemingly chosen in a perfunctory manner believing that they adequately describe their paper and its contents. This illustrates a lack of awareness of the role of these words. In fact key words are those listed in the Medical Subject Heading $(\mathrm{MeSH})$ : the same headings are used in Index Medicus and Medline and are used internationally and across biological disciplines to index scientific data. They cannot be chosen other than by reference to these sources for if they are inconsistent manual and electronic searches for data will fail. This means that without proper key words your article may be lost to posterity!

\section{Ownership}

Many may think these considerations prosaic but they do have importance, as does this possibility of the incursion of fraudulent material. We have to consider that possibility even though we cocoon ourselves into believing that it rarely if ever happens. Nonetheless readers will be conscious of the publicity which has surrounded some high profile cases where fraudulent research has been uncovered or where dishonest claims have been made in the published literature (Lock, 1996). Therefore, in an attempt to ensure that authors are responsible for the claims they make, we intend to enforce two requirements - one of them new - which must be met before a paper is reviewed and published. The first is that authors must disclose any financial relationship which could be construed as a conflict of interest. Such include grant monies, honoraria, fees or gifts related to the work as well as indirect financial support where equipment or drugs have been supplied to facilitate a clinical trial. All potential conflicts of interest must be admitted and all authors should accept responsibility in ensuring that these are divulged. Where none such have occurred then this should also be clearly stated. Secondly, as with other publications, such as the British Medical Journal (Smith, 1997), we are also keen to ensure that all the authors listed on a paper have indeed made some direct contribution to the manuscript. We regularly receive papers with multiple authors some of whom, even in the most 
charitable light, can have had only an extremely peripheral role in data collection or authorship. The obvious examples are single case reports apparently 'written' by five or more authors - which scarcely seems credible. We therefore intend to adopt a system where, in a covering letter, the exact role that has been played by those listed as authors is outlined. Unless otherwise stated the first named author should also be considered as being responsible for the overall content and should sign the covering letter to indicate that he or she is prepared to be held responsible for the integrity of all of its content: where this differs, and another author is assuming the role, this must be made clear. Where affirmation of clear responsibility for a paper is not apparent, or appears dubious, we will reserve the right to deny publication.

\section{Clinical records}

Finally we wish to clarify issues concerning the publication of clinical records (case reports). These have always played an important place in the Journal and we wish to see this tradition continued. However we are concerned that quality of these varies widely. Clinical Records should be concise (no more than four pages of A4 text) and should be confined to single cases without precedent in the world literature or to cases which illustrate some entirely new facet in management or investigation. Reports of relative rarities are also welcome but must add to our understanding of a clinical issue. The reporting of the $\mathrm{n}^{\text {th }}$ case of something which has already been well documented and which, in itself, provides no added value will not be considered.

We are keen to see that the Journal continues to make a contribution. To that end we hope that this editorial will directly help you in drafting material for publication. We urge you, for all the reasons enumerated, to heed the guidelines that have been prepared and the changes that have been made. These changes will also be displayed, in the near future, on the updated version of our Web site which we hope will develop as an adjunct to the written version of the Journal. But whether you read the instructions in written or electronic format is scarcely relevant. What is important is that you tailor your submissions accordingly. At present the production editors have to spend considerable time checking manuscripts and key words and returning papers to authors so that changes can be made. At best this delays publication of your article: at worse it makes it considerably more likely that it will be rejected.

NeIL WeIR

GUY KENYON

\section{References}

Lock, S. (1996) Research misconduct: a resume of recent events. In Fraud and misconduct in medical research (Lock S., Wells, F. eds.). BMJ Publishing, London.

Smith, R. (1997) Authorship: time for a paradigm shift? British Medical Journal 314: 992. 